\title{
Economic Policy Uncertainty and Stock Returns of Africa: A Wavelet Coherence Analysis
}

\author{
Emmanuel Asafo-Adjei $\mathbb{D}^{1},{ }^{1}$ Daniel Agyapong $\mathbb{D}^{1},{ }^{1}$ Samuel Kwaku Agyei $\mathbb{D}$, ${ }^{1}$ \\ Siaw Frimpong $\mathbb{D},{ }^{1}$ Reginald Djimatey $\mathbb{D}^{2},{ }^{2}$ and Anokye M. Adam $\mathbb{D}^{1}$ \\ ${ }^{1}$ Department of Finance, School of Business, University of Cape Coast, Cape Coast, Ghana \\ ${ }^{2}$ Department of Entrepreneurship and Business Sciences, School of Management Sciences and Law, \\ University of Energy and Natural Resources, Sunyani, Ghana \\ Correspondence should be addressed to Emmanuel Asafo-Adjei; eaadjei12998@gmail.com
}

Received 3 September 2020; Revised 4 October 2020; Accepted 5 November 2020; Published 23 November 2020

Academic Editor: Junhai Ma

Copyright ( 92020 Emmanuel Asafo-Adjei et al. This is an open access article distributed under the Creative Commons Attribution License, which permits unrestricted use, distribution, and reproduction in any medium, provided the original work is properly cited.

\begin{abstract}
This study explores how global economic policy uncertainty (EPU) shocks comove with stock returns (SR) of eight African countries-Botswana, Ghana, Kenya, Morocco, Namibia, Nigeria, South Africa, and Zambia. The study employed daily data from December 2010 to December 2019 using wavelet coherence analysis. The results showed that global EPU comoves with most of the SR of African markets and was concentrated in the longer term, especially during the period between 2011 and 2019, although not substantially. The findings indicate that short-term investments in African stocks are less susceptible to global economic policy uncertainty. It is recommended that foreign investors could hedge agaist policy uncertainties by investing in stock listed in African Stock exchanges while appropriate country-level policies are deployed to manage long-term effect of EPU.
\end{abstract}

\section{Introduction}

The role played by economic policy uncertainty in economic activity has aroused interest from researchers, investors, and policymakers since the emergence of the 2008 global financial crisis [1]. There has been growing awareness in understanding the role that economic policy uncertainty plays in motivating macroeconomic fluctuations. In principle, economic policy uncertainty, which includes uncertainty related to fiscal policy, monetary policy, or regulations, can negatively affect the economy [2-5]. Extant literature has postulated that an increase in EPU hurts aggregate investment, the employment rate, and industrial production. Furthermore, many recent empirical studies indicate that EPU shocks in the form suggested by Baker et al. [6] increase stock market turbulence. While many studies confirm this negative influence of domestic policy uncertainty shock on the macroeconomy and equity markets, there is only limited empirical evidence in the literature about the impact of global EPU on stock market returns $[7,8]$.
Several studies have discussed the individual and overall effects of economic policy uncertainty in other sectors such as commodity markets [9], inflation and output [10], economic development [11], exchange rate expectations [12], and comovement of stock markets [13]. Concerning the distinct effects, the behaviours of enterprises are influenced by economic policies, and thus, the operational risks of enterprises increase under EPU and thereby stock performance [14]. Generally, the outcome of these studies is that economic policy uncertainty significantly impacts the usefulness of policy intervention on the economy as a whole, including stock markets.

Handley and Limão [15] showed that trade policy uncertainty plays an important role in firms' investments by utilizing firm-level data. Baker et al. [6] demonstrated that US economic policy uncertainty, measured based on US newspapers, adversely affects production, investment, and employment in the US. Furthermore, Iqbal et al. [16] examined the relationship between economic policy uncertainty (EPU) and firm performance of US-listed nonfinancial firms. Several 
studies have attempted to employ various indicators of EPU to explore its effect on stock market volatility, which mostly focused on country-specific uncertainty on stock returns $[7,8,14,17-19]$. Notwithstanding substantial advances in the related literature, there is limited research that analyses the way global economic policy uncertainty proxy by a major economy EPU affects stock market returns in the African continent. Uncertainty upturns economic disturbance and thus intensifies stock market investment risk. The impact of EPU in major economies on investment risk in the global stock market places can be devastating. The policies of developed economies can result not only in the fizz and failure of emerging and developing countries but also in a global financial crisis. It is, therefore, necessary to understand how uncertainty in the global economic policy impacts developing equity markets.

Within this body of literature, research that concentrates on the EPU-stock return nexus is limited. There is evidence in the literature showing the connectedness and spillover effects of global EPU on stock markets. For example, see Ko and Lee [7], Bernal et al. [20], Dakhlaoui and Aloui [21], Das and Kumar [22], Belke and Osowski [23], and Li et al. [8]. The results of these studies point to a negative relationship between global EPU and stock returns in line with the efficient market and market expectation hypotheses [24, 25]. However, the studies mostly concentrated at developed and important stock markets which are highly integrated into the global financial markets $[7,8]$. Africa stock markets are not only less developed and less effecient but also considered to be generally less integrated into the global financial markets. This suggests that EPU-stock market nexus, as postulated by existing findings on developed economies may be less profound in Africa.

A recent study by Anyikwa and Le Roux [26] shows that African stock market is still segmented from the global, in spite of plunges in the value of most markets in times of global financial crisis and Eurozone sovereign debt crisis. The study, however, observed that the reaction of African stock markets during these crises was mainly driven by the contagion effect, which is driven by the fundamental linkages that exist across markets instead of asset holdings and the behaviour of international investors [27-29]. This characteristic of African stock markets makes the relationship between international EPU and African stock market return an important phenomenon to investigate because of the long-standing belief that African stock markets can provide a diversification opportunity. Adam [30] quantified the information flow from international economic policy uncertainty to African stock markets and observed an asymmetry in the flow of information using transfer entropy. However, the suspicion of evidence of contagion effect of global stock markets on African stock markets requires that the relationship between global EPU and African stock markets are analysed in timefrequency mode. The current study extends the existing literature by examining the effects of global EPU shocks on the African stock market returns. Specifically, this paper examines the time-frequency comovement structure between EPU and selected African stock markets' returns from 2010 to 2019. The paper contributes to the literature on global EPU and SR by examining the time-frequency correlation of global EPU with African stock market returns.
The main contributions of the paper are as follows: first, the study is conducted in the context of Africa since the African stock market is believed to be segmented from the global financial market, irrespective of the drop in the value of most African stock markets returns in times of the global financial crises and the Eurozone sovereign debt crisis as suggested [26].

Again, wavelet analysis decomposes the time series of EPU and SR into the frequency domain to assess whether the timing of global EPU overlaps with the timing of strong comovement in the stock market returns of African countries as suggested. This approach enabled the researchers to simultaneously analyse the comovement at different time scales and different periods to facilitate understanding of the EPU-stock return nexus. Thus, the wavelet analysis helps to understand the mechanism through which EPU comoves with SR and for how long to enable investors as well as policymakers to take economic decisions. Moreover, by analysing the influence of global EPU on each selected African country stock returns, it allows us to obtain the responses of stock returns to EPU for each of the eight countries separately rather than the average response obtained under the standard panel data approach. Furthermore, since EPU shocks have the tendency of impacting international stock returns [31,32], we used global EPU as an indicator of economic policy uncertainty on the stock returns of eight African countries.

The outcome of this study may have significant realworld implications for both policymakers and investors. In the short term (approximately 0-16 days), the EPU-SR nexus is weak in Africa. Thus, global EPU may not be stronger in predicting the future variation of stock markets returns in Africa. As a result, short-term investors could utilize African stocks as a hedge against global uncertainty. However, the comovements strengthened in the long term (approximately 50-256 days) with respect to increases in the period (from 2011-2019). This proves some amount of interacting relationship between global EPU and African stock markets. Thus, investors, particularly those concern with the stock markets in Botswana, Namibia, Morocco, Kenya, South Africa, and possibly Nigeria in the medium and long term, should be vigilant about variations in global EPU. The rest of the paper is structured as follows. The next section addresses the issues of research methodology and Section 3 presents the empirical analysis. The conclusion of the study which includes the implication and recommendations of the findings is presented in Section 4.

\section{Methodology}

The study investigates the time-frequency comovement between global EPU and SR in African stock markets using continuous wavelet and cross-wavelet transforms. There are two types of wavelet transforms, which are continuous wavelet transform (CWTs) and discrete wavelet transforms (DWTs). In the following sections, we discuss only the continuous wavelet transform (CWTs) which was used in this study because it has a better feature extraction purpose as compared to DWT which has noise reduction as well as 
data compression (Pal and Mitra [33], Li et al. [8], and Wu et al. [34]).

2.1. Continuous Wavelet Transform (CWT). In general, a wavelet transform function is used to decompose time series into elementary functions, which are derived from a mother wavelet. The mother wavelet consists of two parameters: time or location $(i)$ and scale (s), which could be defined as follows:

$$
\begin{aligned}
\psi_{i, s}(t) & =\sqrt{s}^{-1} \psi\left(t-i^{\prime}\right)\left(s^{-1}\right), \\
\psi(\cdot) & \in L^{2}(R),
\end{aligned}
$$

where $\sqrt{s}^{-1}$ is the normalization factor, ensuring that the unit variance of the wavelet $\left\|\psi_{i, s}(t)\right\|^{2}=1$; $i$ is the location parameter, providing the exact position of the wavelet; and $s$ is the scale dilation parameter, defining how the wavelet is stretched. Thus, the Morlet wavelet can be defined as follows:

$$
\varphi^{M}(t)=\pi^{-1 / 4} e^{i \omega_{0} t} e^{-t^{2} / 2},
$$

where $\omega_{o}$ is the central frequency of the wavelet. $\omega_{o}$ is set at 6 , following Rua and Nunes [35] and Vacha and Barunik [36].

A time series $x(t)$ with respect to a selected mother wavelet can be decomposed [8] as follows:

$$
w_{x}(\hat{i}, s)=\int_{-\infty}^{\infty} \mathrm{x}(\mathrm{t}) \sqrt{\mathrm{s}}^{-1} \psi\left(\frac{\mathrm{t}-\hat{i}}{\mathrm{~s}}\right) \mathrm{dt} .
$$

By projecting the specific wavelet $\psi(\cdot)$ onto the selected time series, we easily obtain $w_{s}(i, s)$. Correspondingly, the main advantage of a CWT is its ability to decompose and reconstruct the function $x(t) \in L^{2}(\mathrm{R})$ :

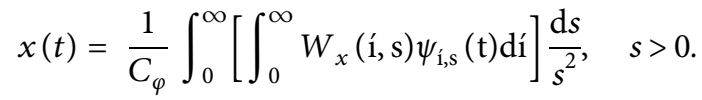

2.2. Wavelet Power Spectrum (WPS). Likened with the classical spectral methods, the WPS can be obtained over a specific time series from the squared absolute value of $w_{x}(i, s),[8]$ defined as follows:

$$
\mathrm{WPS}_{x}(\hat{i}, \mathrm{~s})=\left[\mathrm{w}_{\mathrm{x}}(\hat{i}, \mathrm{~s})\right]^{2} .
$$

2.3. Wavelet Coherence. The WPS has some limitations in low-frequency oscillations [37]. To reduce the bias of WPS, we use the cross-wavelet transform tool developed by $\mathrm{Ng}$ and Chan [38] to analyse the dependence structure between EPU and stock returns of African stock market. The use of wavelet coherence is well structured in the extant literature (see Junior et al. [39], Wu et al. [34], and $\mathrm{Li}$ et al. [8]). The cross-wavelet transform describing covariance in the timefrequency domain is defined as follows:

$$
W_{x y}=W_{x}(i, \mathrm{~s}) \mathrm{W}_{\mathrm{y}}^{*}(i, \mathrm{~s}),
$$

where $W_{x}(i, \mathrm{~s})$ and $W_{y}^{*}(i, \mathrm{~s})$ denote the cross-wavelet of series $x(t)$ and $y(t)$, respectively (see Torrence and Compo
[40] and Li et al. [8]). * indicates a complex conjugate. The cross-wavelet transform shows the area in time space with high common power.

WTC is well defined as the squared absolute value of normalizing a wavelet cross-spectrum to a single wavelet power spectrum [41]. As a result, the squared wavelet coefficient is expressed as follows:

$$
R^{2}(x, y)=\frac{\left|\rho\left(s^{-1} W_{x y}(\mathrm{i}, \mathrm{s})\right)\right|^{2}}{\rho\left(s^{-1}\left|W_{x}(\mathrm{i}, \mathrm{s})\right|^{2}\right) \rho\left(s^{-1}\left|W_{y}(\mathrm{i}, \mathrm{s})\right|^{2}\right)},
$$

where $\rho$ indicates a smoothing factor, which balances resolution and significance, and $0 \leq R_{\mathrm{xy}}^{2}(\mathrm{i}, \mathrm{s}) \leq 1$. A value close to 0 specifies a weak relationship, while a value close to 1 indicates a strong relationship. There is a complete comovement between the series in the time-frequency domain depicted by wavelet analysis. A stronger correlation or dependency is demonstrated by a hotter colour. The statistical significance of the coherence is inspected by the Monte Carlo procedure since the theoretical distribution of the cross-wavelet transforms coefficient is unknown (see Torrence and Compo [40] and Li et al. [8]). Furthermore, the bias problem in the wavelet power spectrum and wavelet cross-spectrum is eliminated by the normalizing function of the wavelet coherence, and PWC and MWC are of no exception. Coherence may be appropriately utilized to scrutinize comovement in international stock markets.

2.4. WTC Phase Difference. The wavelet transform coherence phase difference indicates the interruptions in the oscillation concerning the examined time series. We assume that $\phi_{x y}$ describes the phase difference in international stock markets. Therefore, following Bloomfield et al. [42], Li et al. [8], and Wu et al. [34], the phase difference between $x(t)$ and $y(t)$ is represented as follows:

$$
\varnothing_{x y}(\mathrm{i}, \mathrm{s})=\tan ^{-1}\left(\frac{\mathfrak{\Im}\left\{\mathrm{S}\left(\mathrm{s}^{-1} \mathrm{~W}_{\mathrm{xy}}(\mathrm{i}, \mathrm{s})\right)\right\}}{\mathfrak{R}\left\{\mathrm{S}\left(\mathrm{s}^{-1} \mathrm{~W}_{\mathrm{xy}}(\mathrm{i}, \mathrm{s})\right)\right\}}\right),
$$

where $\mathfrak{I}$ and $\mathfrak{R}$ are the imaginary operators and real operator, respectively. In the wavelet coherence map, the dimensional phase pattern defines the effects of the wavelet coherence difference. The dimensional arrows are used to distinguish different phase patterns. For instance, if $x(t)$ and $y(t)$ are in phase, the arrow points to the rightward (or leftward). Likewise, if the arrow points downward (or upward), this implies that $y(t)$ or $x(t)$ is leading.

\section{Empirical Analysis}

3.1. Data. The data used for the analysis consist of daily stock returns of eight stock markets in Africa made up of Ghana, Nigeria, Zambia, Namibia, Morocco, Kenya, Botswana, and South Africa and global EPU spanning from December 2010 to December 2019. A total of 2,352 observations were used for the study. The suggested period was chosen to minimize the effect of the financial crisis that ended around June 2009, and to ensure an equitable 
comparison after the recovery of the global economy from the crisis but covers European Sovereign debt crises, USChina trade tension, Brexit, and the emergence of COVID19 as a global pandemic. We seek to ascertain if EPU comoves with African stock markets after the emergence of the financial crisis. The countries were selected based on data availability for the chosen periods, yet it contains most of the important markets in Africa. Daily data were selected over monthly series because daily data utilize better-off information over the monthly dataset (see Bannigidadmath and Narayan [43] and Das and Kumar [22]). The EPU developed by Baker et al. [6] was obtained from the website https:// www.policyuncertainty.com/index.html and SR from the African stock exchange. The analysis was based on the returns of daily indexes as shown in the following equation:

$$
r_{t}=\ln P_{t}-\ln P_{t-1}
$$

where $r_{t}$ is the continuously compounded return and $P_{t}$ and $P_{t-1}$ are current and previous indexes, respectively.

3.2. Descriptive Statistics. Figure 1 shows the graphical representation of the time series plot of both indexes and returns of the EPU and the eight African stock markets considered in this study. An informal stationarity test was done by analysing the trend of the indexes and returns used in the study. A careful look at the plots indicates that most indexes are trending downwards, which suggests that these series are nonstationary. They are seen to be $I(1)$, that is, they have stochastic trends. This depicts that some stock markets in Africa after the recent global financial crisis have been performing not as much as previously.

Again, it could be seen that the returns seem to follow the same trend. After the first difference of all the variables, they become stationary as they revert around zero as shown in Figure 1.

Table 1 depicts the descriptive statistics of EPU and SRs of the eight African countries for this study. Almost all returns had negative means except for EPU and Kenya. Thus, the performance of the SRs requires immediate attention by policymakers to restore them to an appropriate level desired by existing and potential investors. Again, aside EPU and SR of Zambia, all the other returns were negatively skewed. This suggests that the stock markets with the negatively skewed SRs of those countries should proceed with caution since there is a potential for repeated lessor gains. It could further be observed that all the datasets are nonnormally distributed.

3.3. Main Results. To ensure the smooth interpretation of the data, right-pointing arrows and left-pointing arrows indicate when EPU and SR variables are in phase (movement in the same direction) and antiphase (movements in the reverse direction), respectively. Right-pointing upward arrows and left-pointing downward arrows indicate that the first variable is lagging, while the left-pointing upward arrows and the right-pointing downward arrows mean that the first variable is leading. The strength of the interdependence between the paired series is indicated by the colour of the surface and depicted by the colour pallet. The red colour (warm) denotes sections with significant interactions, while blue colour (cold) indicates a lower correlation between the series.

The output in Figure 2 depicts the wavelet analysis of EPU and SR of selected African markets. Generally, there is not much coherency between EPU and SR in African markets. However, there were periods where EPU interrelated with SR, but only in the long term. It is therefore imperative to interpret the EPU shocks on SR from a timefrequency domain.

In the short term (approximately 0-16 days), little can be said of the coherency between global EPU and SR as the comovement is weak throughout the period (i.e., from 2011 to 2019) for all countries. A quick scan of the coherency between EPU and SR in most countries shows that coherency increases with time (i.e., from 2011 to 2019), but only in the long term except for Ghana and Zambia. Over approximately 250 days, investors who held their investments (from 2011 to 2013) in countries such as Botswana, Kenya, Nigeria, Zambia, and possibly Ghana were likely to experience the impact of global EPU on their returns. The leftpointing upward arrows in the case of Botswana, Ghana, and Nigeria for this period signify that EPU was leading, while the right-pointing upward arrows and left-pointing downward arrows in the case of Kenya and Zambia, respectively, indicate that SR was leading. This result is consistent with the study of $\mathrm{Li}$ et al. [8], who revealed that the interaction between EPU in the US and stock returns in China and India is weak in the short term but gradually becomes stronger in the long term.

Again, from 2014 to 2015 (approximately 20-70 days), the interrelation strengthened in countries such as Botswana, Ghana, Namibia, and South Africa simultaneously. The left-pointing upward arrows indicate that EPU was the leading variable in this period. This suggests that SRs in these countries were lagging (approximately 2014-2015). It can, however, be said that the markets during this period may have some commonalities which may be suggested for further research.

Furthermore, it could be examined that from 2017 to 2019, Botswana, Kenya, Morocco, Namibia, Nigeria, and South Africa experienced some level of comovements with global EPU and SR in the medium to long term (approximately more than 50 days). The left-pointing arrows for the six countries during this period demonstrate inverse comovements between global EPU and SR. There are patches of similarities within the stock market of these countries which may require further analysis. This is consistent with the results of Ko and Lee [7], who investigated the comovement between economic policy uncertainty and stock price in both time and frequency domains and revealed that the relationship is generally negative but fluctuates overtime displaying low- to high-frequency cycles. Additionally, the similarities of this study include the results of Tsai [14] who explored the effect of EPU in four countries on the contagion risk of investments in the global stock market, which revealed that EPU in China and its contagion risk spreads to different regional markets. 

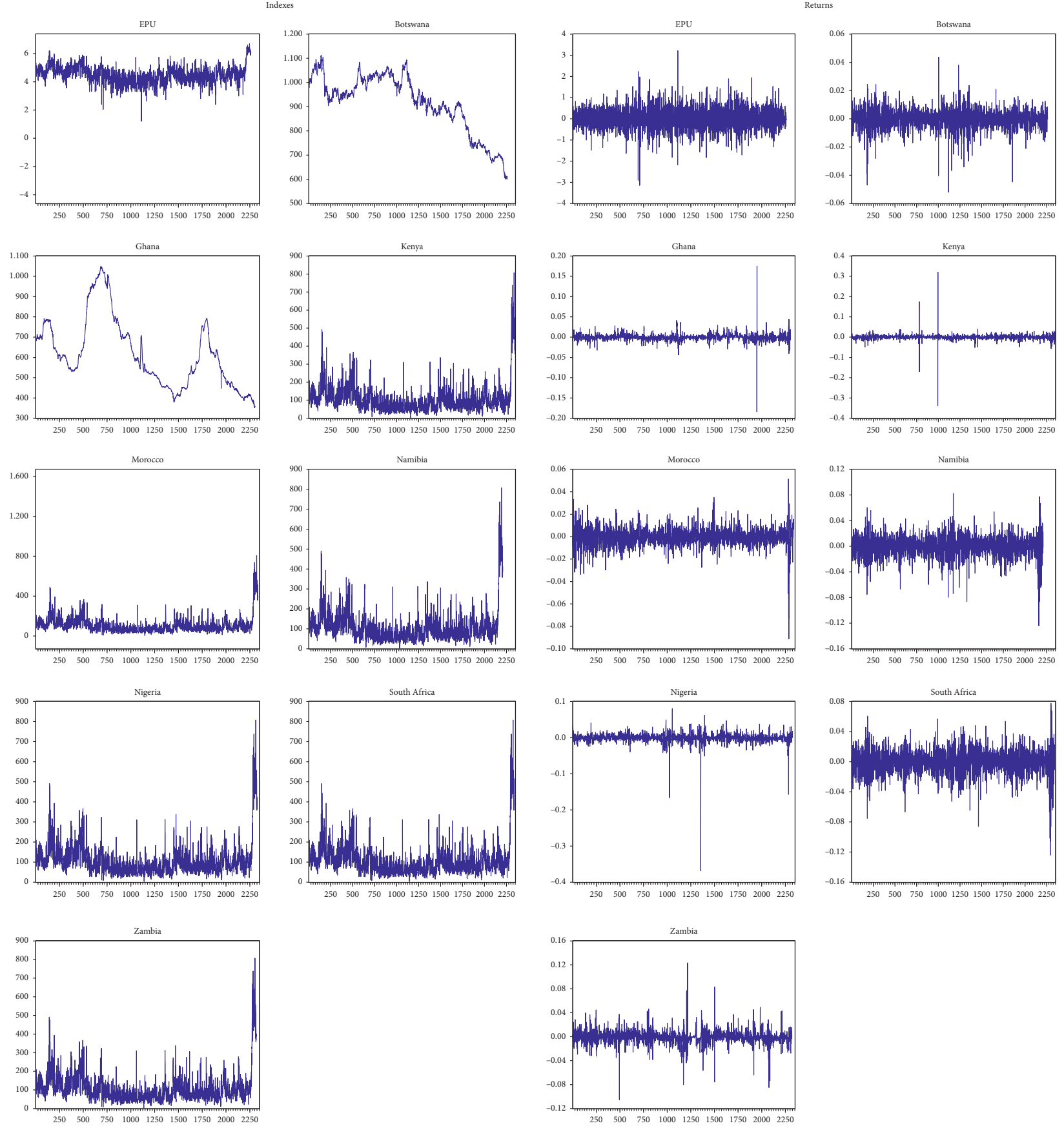

FIGURE 1: Graphical representation of returns of time series data.

TABLE 1: Descriptive statistics of EPU and SR.

\begin{tabular}{|c|c|c|c|c|c|c|}
\hline Countries/EPU & Mean & Std. dev. & Skewness & Kurtosis & Jarque-Bera & Observations \\
\hline Botswana & -0.0002 & 0.0070 & -0.6501 & 9.0650 & $3624.594^{* * *}$ & 2352 \\
\hline Ghana & -0.0003 & 0.0090 & -0.3960 & 142.4446 & $1867571^{* * *}$ & 2352 \\
\hline Kenya & 0.0000 & 0.0140 & -1.0110 & 283.7185 & $7723081^{* * *}$ & 2352 \\
\hline Morocco & -0.0002 & 0.0081 & -0.9977 & 15.4287 & $15429.55^{* * *}$ & 2352 \\
\hline Namibia & -0.0002 & 0.0168 & -0.6927 & 7.9385 & $2421.498^{* * *}$ & 2352 \\
\hline Nigeria & -0.0004 & 0.0136 & -9.7755 & 249.1509 & $5911792^{* * *}$ & 2352 \\
\hline South Africa & -0.0002 & 0.0163 & -0.6712 & 7.7351 & $2362.741^{* * *}$ & 2352 \\
\hline Zambia & -0.0005 & 0.0116 & 0.1078 & 19.6097 & $26661.65^{* * *}$ & 2352 \\
\hline EPU & 0.0001 & 0.5087 & 0.0194 & 5.5119 & $594.5533^{* * *}$ & 2352 \\
\hline
\end{tabular}




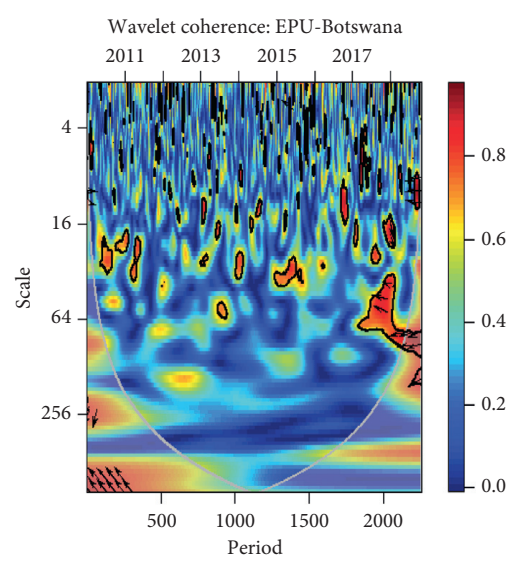

(a)

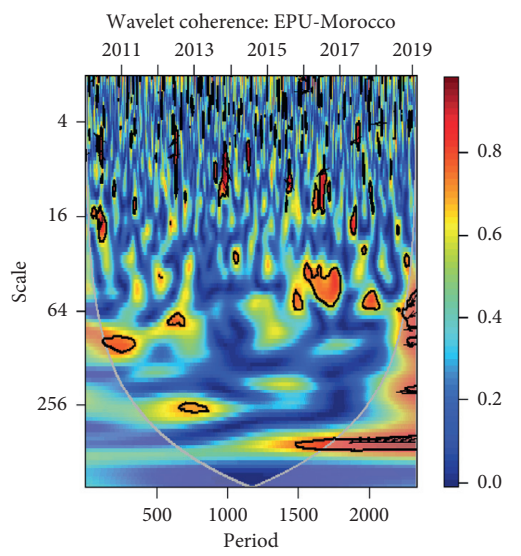

(d)

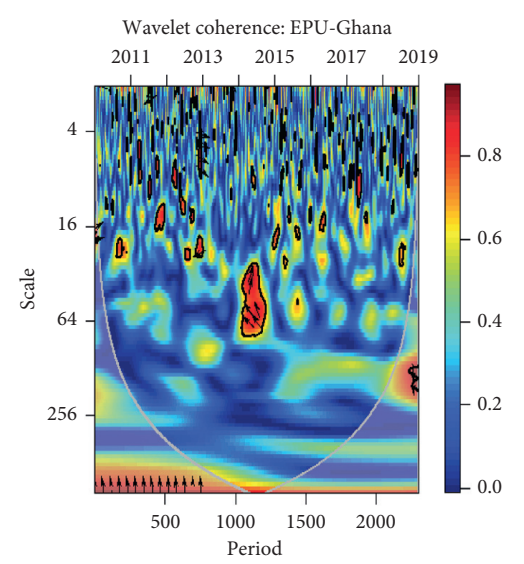

(b)

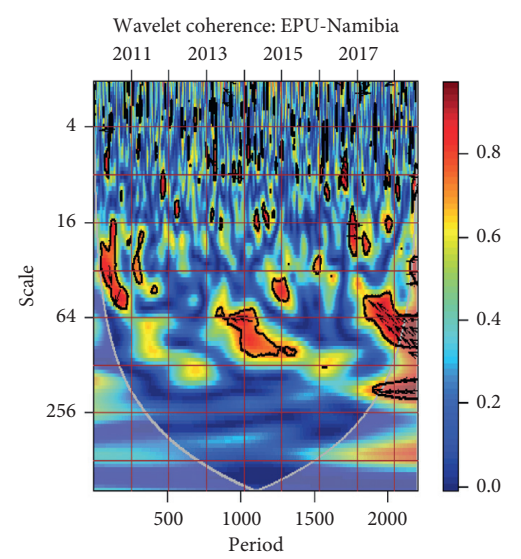

(e)

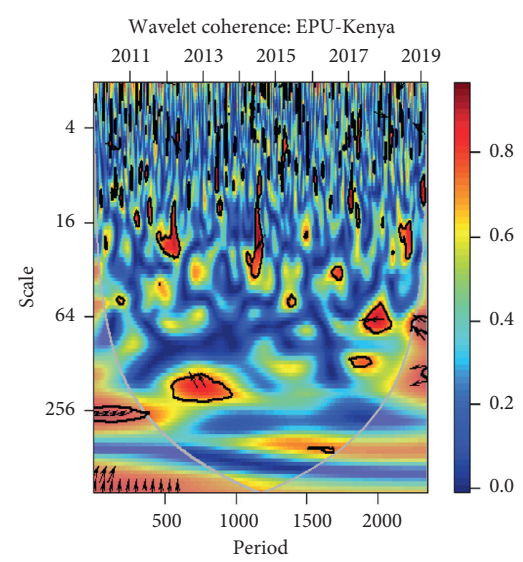

(c)

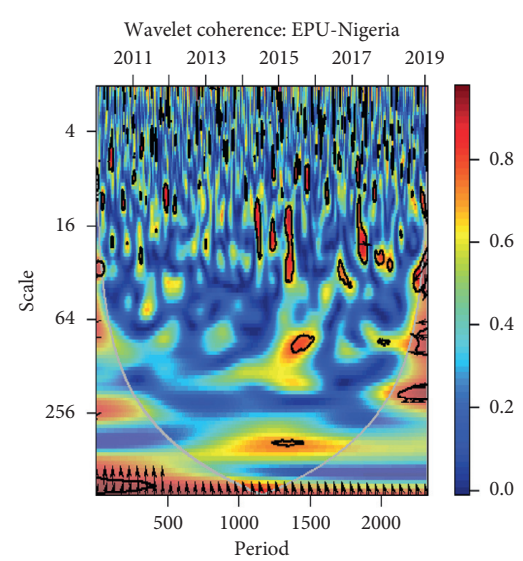

(f)

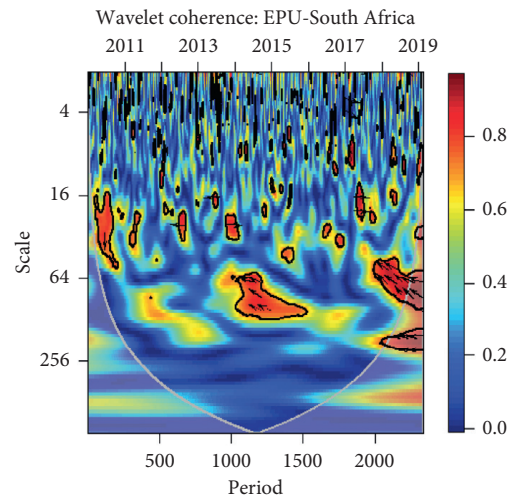

(g)

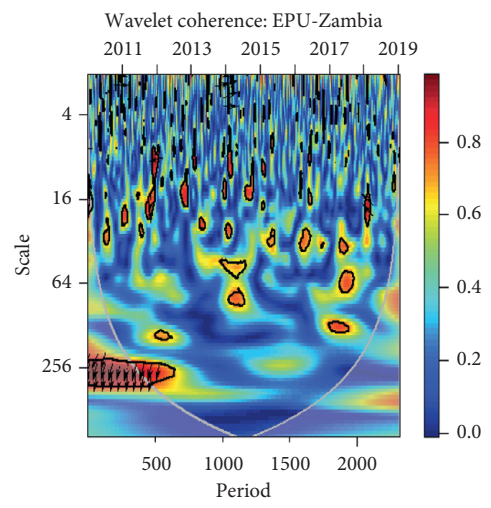

(h)

FIgURE 2: Wavelet analysis of EPU and African SR.

\section{Conclusion}

This paper examines the relationship between EPU and SR based on wavelet analysis by utilizing daily data on the African stock market. We use the Baker et al. [6] measure for eight African countries for the years from December 2010 to December 2019.

Our results and conclusions reveal that the continuous wavelet coherence depicts a weak comovement between global EPU and stock markets in Africa in the short term (approximately 0-16 days) from 2011 to 2019. However, the comovements fortify in the long term (approximately 50 days), especially concerning increases in periods. Specifically, global EPU comoves with African stock markets in the medium to long term. This finding is consistent with the view that the impact of global EPU on African stock markets is mainly noticeable, increasing with time in the long term after the 2008 global financial crisis. However, right after the crisis, the effects of the global EPU seem to have a very partial response to stock markets in Africa (for less than 250 days) in 2011.But, in the long term (over 250 days), Botswana, Ghana, Kenya, Nigeria, and Zambia saw comovement with 
global EPU and SR right after the global financial crisis (approximately 2011-2013).

The evidence offered in this study may have significant real-world implications for both policymakers and investors. In the short term (approximately 0-16 days), the comovement is weak. Global EPU may not be robust to predict the future variation of stock markets in Africa. As a result, shortterm investors could utilize African stocks as a hedge against global uncertainty. The fortified comovement in the long term (approximately 50-256 days) with respect to increases in period (from 2011-2019) justifies some amount of interacting relationship between the global EPU and African stock markets. Thus, investors, particularly those with concern with the stock markets in Botswana, Namibia, Morocco, Kenya, South Africa, and possibly Nigeria in the medium and long term, should be vigilant about variations in global EPU. In this regard, since the changes of global EPU may obstruct the investors' confidence in stock markets, African policymakers need to reflect on the long-term development of stock markets and also judiciously fine-tune policies to better direct short-term investors.

\section{Data Availability}

The stock return and economic policy uncertainty data were supplied by Datastream and economic policy uncertainty is under license and so cannot be made freely available.

\section{Conflicts of Interest}

The authors declare that they have no conflicts of interest.

\section{References}

[1] X. Sun, X. Chen, J. Wang, and J. Li, "Multi-scale interactions between economic policy Uncertainty and oil prices in timefrequency domains," The North American Journal of Economics and Finance, vol. 51, Article ID 100854, 2020.

[2] V. Colombo, "Economic policy uncertainty in the US: does it matter for the Euro area?" Economics Letters, vol. 121, no. 1, pp. 39-42, 2013.

[3] S. R. Henzel and M. Rengel, "Dimensions of macroeconomic uncertainty: a common factor analysis," Economic Inquiry, vol. 55, no. 2, pp. 843-877, 2017.

[4] P. Meinen and O. Roehe, "On measuring uncertainty and its impact on investment: cross-country evidence from the euro area," European Economic Review, vol. 92, pp. 161-179, 2017.

[5] Y. Kido, "The transmission of US economic policy uncertainty shocks to Asian and global financial markets," The North American Journal of Economics and Finance, vol. 46, pp. 222-231, 2018.

[6] S. R. Baker, N. Bloom, and S. J. Davis, "Measuring economic policy uncertainty*," The Quarterly Journal of Economics, vol. 131, no. 4, pp. 1593-1636, 2016.

[7] J.-H. Ko and C.-M. Lee, "International economic policy uncertainty and stock prices: wavelet approach," Economics Letters, vol. 134, pp. 118-122, 2015.

[8] R. Li, S. Li, D. Yuan, and K. Yu, "Does economic policy uncertainty in the US influence stock markets in China and India? time-frequency evidence," Applied Economics, vol. 52, no. 39, pp. 1-17, 2020.
[9] Y. Wang, B. Zhang, X. Diao, and C. Wu, "Commodity price changes and the predictability of economic policy uncertainty," Economics Letters, vol. 127, pp. 39-42, 2015.

[10] P. M. Jones and E. Olson, "The time-varying correlation between uncertainty, output, and inflation: evidence from a DCC-GARCH model," Economics Letters, vol. 118, no. 1, pp. 33-37, 2013.

[11] E. M. Scheffel, "Accounting for the political uncertainty factor," Journal of Applied Econometrics, vol. 31, no. 6, pp. 11048-11064, 2016.

[12] J. Beckmann and R. Czudaj, "Exchange rate expectations and economic policy uncertainty," European Journal of Political Economy, vol. 47, pp. 148-162, 2017.

[13] X.-M. Li and L. Peng, "US economic policy uncertainty and co-movements between Chinese and US stock markets," Economic Modelling, vol. 61, pp. 27-39, 2017.

[14] I.-C. Tsai, "The source of global stock market risk: a viewpoint of economic policy uncertainty," Economic Modelling, vol. 60, pp. 122-131, 2017.

[15] K. Handley and N. Limão, "Trade and investment under policy uncertainty: theory and firm evidence," American Economic Journal: Economic Policy, vol. 7, no. 4, pp. 189-222, 2015.

[16] U. Iqbal, C. Gan, and M. Nadeem, "Economic policy uncertainty and firm performance," Applied Economics Letters, vol. 27, no. 10, pp. 765-770, 2020.

[17] L. Liu and T. Zhang, "Economic policy uncertainty and stock market volatility," Finance Research Letters, vol. 15, pp. 99105, 2015.

[18] P. Alessandri and H. Mumtaz, "Financial regimes and uncertainty shocks," Journal of Monetary Economics, vol. 101, pp. 31-46, 2019.

[19] I. O. Olanipekun, G. Olasehinde-Williams, and H. Güngör, "Impact of economic policy uncertainty on exchange market pressure," Sage Open, vol. 9, no. 3, 2019.

[20] O. Bernal, J.-Y. Gnabo, and G. Guilmin, "Economic policy uncertainty and risk spillovers in the Eurozone," Journal of International Money and Finance, vol. 65, pp. 24-45, 2016.

[21] I. Dakhlaoui and C. Aloui, "The interactive relationship between the US economic policy uncertainty and BRIC stock markets," International Economics, vol. 146, pp. 141-157, 2016.

[22] D. Das and S. B. Kumar, "International economic policy uncertainty and stock prices revisited: multiple and Partial wavelet approach," Economics Letters, vol. 164, pp. 100-108, 2018.

[23] A. Belke and T. Osowski, "International effects of euro area versus U.S. Policy uncertainty: a favar approach," Economic Inquiry, vol. 57, no. 1, pp. 453-481, 2019.

[24] M. Bahmani-Oskooee and S. Saha, "On the effects of policy uncertainty on stock prices: an asymmetric analysis," Quantitative Finance and Economics, vol. 3, no. 2, pp. 412424, 2019.

[25] T. C. Chiang, "Market efficiency and news dynamics: evidence from International equity markets," Economies, vol. 7, no. 1, 2019.

[26] I. Anyikwa and P. Le Roux, "Integration of African stock markets with the developed stock markets: an analysis of comovements, volatility and contagion," International Economic Journal, vol. 34, no. 2, pp. 279-296, 2020.

[27] O. Hemche, F. Jawadi, S. B. Maliki, and A. I. Cheffou, "On the study of contagion in the context of the subprime crisis: a dynamic conditional correlation-multivariate GARCH approach," Economic Modelling, vol. 52, pp. 292-299, 2016. 
[28] S. Celik, "The more contagion effect on emerging markets: the evidence of DCC GARCH model," Economic Modelling, vol. 29, no. 5, pp. 1946-1959, 2012.

[29] B. H. Boyer, T. Kumagai, and K. Yuan, "How do crises spread? evidence from accessible and inaccessible stock indices," The Journal of Finance, vol. 61, no. 2, pp. 957-1003, 2006.

[30] A. M. Adam, "Susceptibility of stock market returns to international economic policy evidence from effective transfer entropy of Africa with the implication for open innovation," Journal of Open Innovation: Technology, Market, and Complexity, vol. 6, no. 3, p. 71, 2020.

[31] L. Yin and L. Han, "Spillovers of macroeconomic uncertainty among major Economies," Applied Economics Letters, vol. 21, no. 13 , pp. 938-944, 2014.

[32] C. Christou, J. Cunado, R. Gupta, and C. Hassapis, "Economic policy uncertainty and stock market returns in Pacific Rim countries: evidence based on a Bayesian panel VAR model," Journal of Multinational Financial Management, vol. 40, pp. 92-102, 2017

[33] D. Pal and S. K. Mitra, "Oil price and automobile stock return co-movement: a wavelet coherence analysis," Economic Modelling, vol. 76, pp. 172-181, 2019.

[34] K. Wu, J. Zhu, M. Xu, and L. Yang, "Can crude oil drive the co-movement in the international stock market? evidence from partial wavelet coherence analysis," The North American Journal of Economics and Finance, vol. 53, Article ID 101194, 2020.

[35] A. Rua and 1. C. Nunes, "International comovement of stock market returns: a wavelet analysis," Journal of Empirical Finance, vol. 16, no. 4, pp. 632-639, 2009.

[36] L. Vacha and J. Barunik, "Co-movement of energy commodities revisited: evidence from wavelet coherence analysis," Energy Economics, vol. 34, no. 1, pp. 241-247, 2012.

[37] Z. Liu, Y. Ye, F. Ma, and J. Liu, "Can economic policy uncertainty help to forecast the volatility: a multifractal perspective," Physica A: Statistical Mechanics and Its Applications, vol. 482, pp. 181-188, 2017.

[38] E. K. W. Ng and J. C. L. Chan, "Geophysical applications of partial wavelet coherence and multiple wavelet coherence," Journal of Atmospheric and Oceanic Technology, vol. 29, no. 12, pp. 1845-1853, 2012.

[39] P. O. Junior, A. M. Adam, and G. Tweneboah, "Co-movement of real exchange rates in the West African monetary zone," Cogent Economics and Finance, vol. 5, no. 1, p. 1351807, 2017.

[40] C. Torrence and G. P. Compo, "A practical guide to wavelet analysis," Bulletin of the American Meteorological Society, vol. 79, no. 1, pp. 61-78, 1998.

[41] A. Grinsted, J. C. Moore, and S. Jevrejeva, Application of the cross wavelet transform and wavelet coherence to geophysical time series, 2004.

[42] D. S. Bloomfield, R. T. J. McAteer, B. W. Lites, P. G. Judge, M. Mathioudakis, and F. P. Keenan, "Wavelet phase coherence analysis: application to a quiet-sun magnetic element," The Astrophysical Journal, vol. 617, no. 1, p. 623, 2004.

[43] D. Bannigidadmath and P. K. Narayan, "Stock return predictability and determinants of predictability and profits," Emerging Markets Review, vol. 26, pp. 153-173, 2016. 